\section{Seguridad y eficacia de una vacuna atenuada contra la gastroenteritis grave por rotavirus}

La infección por rotavirus es la principal causa de enfermedad y muerte por diarreas en lactantes y niños menores. El desarrollo de una vacuna segura y eficaz contra el rotavirus es de la mayor prioridad, especialmente - aunque no únicamentepara los países en desarrollo, donde es mayor la carga de infección.

Estudios previos han demostrado que la vacuna atenuada contra el rotavirus humano elaborada con la cepa RIX4414 de especificidad G1P[8] (Rotarix, GlaxoSmithKline Biologicals) es segura e inmunogénica y que su eficacia contra la gastroenteritis grave por rotavirus es de 90 a $100 \%$. La protección comenzó desde la primera dosis de la vacuna y se extendió hasta que los niños cumplieron 2 años, tanto contra los rotavirus del grupo G1P[8] como contra los del grupo G9P[8].

El objetivo del presente trabajo fue confirmar la seguridad y la eficacia de esta vacuna mediante un ensayo clínico de fase III, multinacional, aleatorizado, con doble enmascaramiento y controlado con placebo.

Investigadores de Argentina, Brasil, Chile, Colombia, Finlandia, Honduras, México, Nicaragua, Panamá, Perú, República Dominicana y Venezuela captaron un total de 63225 niños sanos de 6 a 13 semanas de edad en clínicas y hospitales públicos de esos países. Los niños participantes se asignaron de manera aleatoria a dos grupos que recibieron respectivamente dos dosis orales de la vacuna evaluada (31 673 niños) o de placebo (31 552 niños) con un intervalo de dos meses. El seguimiento fue de 100 días (mediana) a partir de la primera dosis. De esa cohorte se seleccionaron los primeros 20169 niños vacunados (10 159 con la vacuna y 10010 con placebo) para hacer el análisis de la eficacia con un seguimiento de 9 a 10 meses. Los casos de gastroenteritis grave se detectaron mediante el seguimiento activo y se clasificaron según la escala de 20 puntos de Vesikari.

La eficacia de la vacuna para prevenir la gastroenteritis grave por rotavirus fue de $85 \%$ con respecto al grupo de placebo $(P<0,001)$ y se elevó a $100 \%$ cuando se tomaron en cuenta solo los casos más graves. La vacuna también se mostró segura con respecto al riesgo de invaginación intestinal.

La hospitalización por diarreas, independientemente de su causa, se redujo en $42 \%$ (intervalo de confianza de 95\% [IC95\%]: 29 a 53\%; P<0,001). La diferencia en el riesgo de invaginación intestinal entre los vacunados y los que recibieron placebo fue de $-0,32$ por 10000 niños (IC95\%: $-2,91$ a 2,18; $P=0,78$ ).

Los resultados obtenidos confirman la seguridad y la eficacia de la vacuna evaluada y su valor para la salud pública. La aplicación de dos dosis orales de esta vacuna protegió a los niños contra la gastroenteritis grave por rotavirus, redujo significativamente la tasa de casos de gastroenteritis por cualquier causa y no incrementó el riesgo de invaginación intestinal en los vacunados. (ClinicalTrials. gov numbers, NCT00139347 and NCT00263666). (Ruiz-Palacios GM et al. Safety and efficacy of an attenuated vaccine against severe rotavirus gastroenteritis. N Engl J Med. 2006;354(1): 11-22.)

\section{Efecto sinérgico del nivel educacional de los padres y la situación económica de la familia en la salud infantil en América Latina}

Diversas investigaciones han demostrado la enorme importancia que tienen los factores socioeconómicos para la salud infantil. Sin embargo, no se conoce si el nivel educacional de los padres y la situación económica de la familia tienen un efecto sinérgico o independiente sobre el riesgo de enfermar o morir de los niños. Si estos factores actuaran de forma sinérgica, las intervenciones dirigidas a mejorar la educación de la población tendrían un menor efecto sobre el mejoramiento de la salud de los niños en ausencia de programas simultáneos de desarrollo económico.

El objetivo de este trabajo fue evaluar la posible acción independiente y combinada del nivel educacional de los padres y la situación económica de la familia sobre el riesgo de sufrir enfermedades diarreicas y respiratorias de los niños menores de 5 años, tanto antes como después de ajustes en función de los principales factores de confusión.

Se analizaron 9 encuestas nacionales de medición de estándar de vida (ENMEV) y 12 encuestas de demografía y salud (EDS) realizadas en ocho países latinoamericanos (Bolivia, Colombia, Ecuador, Guatemala, Nicaragua, Panamá, Perú y República Dominicana).

Se tomaron en cuenta determinantes proximales (la contaminación ambiental, la nutrición, la 